\title{
Predictive Model of Cerebrospinal Fluid Leakage After Posterior Circumferential Decompression for Thoracic Ossification of Posterior Longitudinal Ligament
}

Jun Zhong

Peking University International Hospital

Bingtao Wen ( $\square$ wenbingtao@pkuih.edu.cn )

Peking University International Hospital

Zhongqiang Chen

Peking University International Hospital

\section{Research Article}

Keywords: cerebrospinal fluid leakage, Circumferential decompression, Ossification of posterior longitudinal ligament, Thoracic, Predictive model

Posted Date: June 10th, 2021

DOl: https://doi.org/10.21203/rs.3.rs-580487/v1

License: (c) (1) This work is licensed under a Creative Commons Attribution 4.0 International License.

Read Full License 


\section{Abstract}

Background: Cerebrospinal fluid leakage(CSFL) is one of the most common complications after posterior transarticular osteotomy and circumferential decompression for thoracic ossification of posterior longitudinal ligament(OPLL). It is of great usefulness If the cerebrospinal fluid leakage can be predicted preoperatively. These predictors help to attract the attention of the surgeon in advance and warn the patient. Therefore, the aim of this study is to find out the factors that can predict the CSFL prior to operation and try to build a predictive model.

Methods: A total of 61 patients with thoracic OPLL underwent posterior transarticular osteotomy and circumferential decompression from August 2015 to June 2020 in our hospital were included in this study, including 29 males and 32 females. The patients were divided into CSLF group and non-CSFL group according to whether they suffered cerebrospinal fluid leakage. Univariate analysis was used to identify possible predictors in Demographic characteristics, clinical and radiological data. A logistic regression model was developed by multivariate analyses to predict probability of CSFL. Model validation was done using the receiver operating characteristic(ROC) curve.

Results: The incidence of CSFL was $31.1 \%$, including 7 males and 12 females, with an average age of $49.8 \pm 11.4$ years. The mean drainage indwelling time in CSFL group was $5.6 \pm 1.0$ days, which was significantly higher than that in non-CSFL group ( $4.2 \pm 1.3$ days, $\mathrm{P}<0.001)$. The mean length of hospital stay was $16.3 \pm 6.3$ days, slightly higher than that of the non-CSF group (15.8 \pm 6.7 days), but there was no statistical difference $(P=0.77)$. Among them, 12 patients $(63.2 \%)$ suffered low intracranial pressure manifested as headache; 1 patient $(5.3 \%)$ had cerebrospinal fluid outflow from the incision, and the wound healed successfully after debridement. 1 patient (5.3\%) was re-admitted to the hospital due to fever after 3 weeks, considering deep wound effusion and pleural effusion. The wound effusion was found to be cured after 2 weeks of anti-infective treatment. Univariate regression analysis showed statistical differences $(\mathrm{P}<0.05)$ in smoking history, segment of circumferential decompression, combined with ossification of the ligamentum flavum (OLF), number of laminectomy, occupying ratio and OPLL base ratio. Multivariate regression model showed smoking history (OR=30.1, $P=0.003)$, the upper thoracic segment $(\mathrm{OR}=188.0, \mathrm{P}=0.002)$, the middle thoracic segment $(\mathrm{OR}=57.4, P=0.005)$ and $\mathrm{OPLL}$ base ratio ( $\mathrm{OR}=1.3, P=0.007)$ were the predictors of CSFL. The ROC curve was in the upper left corner (area under the curve $=0.955,95 \% \mathrm{Cl} 0.91-1.00, \mathrm{P}<0.001)$, indicating good predictability of the model.

Conclusion: The predictive model established in this study has a high predictive effect. When the patients with thoracic OPLL have smoking history or the segment of circumferential decompression is located in the upper or middle thoracic spine or the OPLL has a wide base, the operator should be highly alert to the possibility of postoperative CSFL and warn the patient before surgery.

Evidence level: level II-2

\section{Background}


The incidence of ossification of the posterior longitudinal ligament (OPLL) in thoracic spine is about $0.8 \%$ [, which is lower than that of the OPLL in cervical spine. However, thorax limits spinal movement and bone compression is static compression. Once clinical symptom occurs, they progress rapidly and can cause severe spinal cord injury or even paraplegia in a short time. Surgery is the only way to treat OPLL of the thoracic spine. Posterior transarticular osteotomy and circumferential decompression can achieve front-and-rear decompression through simple posterior approach. It can both avoid surgical complications of anterior approach and achieve direct decompression. Many scholars have confirmed that this operation can achieve satisfactory surgical results []. However, the operation is highly difficulty and has many complications, among which cerebrospinal fluid leakage (CSFL) is the most common one 7. This complication increases the risk of postoperative infection [, even leads to central nervous system infection and long-term formation cerebrospinal fluid pseudocysts []. At present, dural ossification is considered to be the main cause of intraoperative dural injury. Nevertheless, neither CT nor MRI can be used to determine whether there is dural ossification in advance. It is not until the operation is implemented can dural ossification be confirmed. So, are there some preoperative factors that can be used to predict the CSFL? These factors help to attract the attention of the surgeon in advance and warn the patient. Therefore, the aim of this study is to find out the factors that can predict the CSFL prior to operation and try to establish a predictive model.

\section{Method}

Patient screening and selection

The cases enrolled in this study were patients who were diagnosed with OPLL in thoracic spine from August 2015 to June 2020 in our hospital. The inclusion criteria included: (1) Age $\geq 18$ years old; (2) The surgery plan was a simple posterior approach to transarticular osteotomy and circumferential decompression; (3) The cases underwent complete preoperative CT examination to determine the type of OPLL and whether there was ossification of the ligamentum flavum (OLF); (4) The surgery was done by

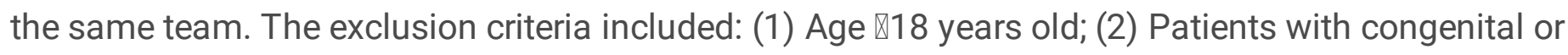
acquired deformities of thoracic spine, such as congenital thoracic scoliosis, ankylosing spondylitis, and kyphosis secondary to spinal tuberculosis; (3) Thoracic infectious diseases; (4) Primary or metastatic spinal tumors; (5) Previous history of thoracic surgery. According to the above inclusion criteria and exclusion criteria, a total of 61 patients were included in this study. Among them, 29 were males and 32 were females. The age range is $27-82$ years (53.4 \pm 13.0 years), and the course ranges from (from clinical symptoms to surgery) $1-36$ months ( $9.2 \pm 8.6$ months) .

Surgical procedure and treatment of dural defect

All patients underwent transarticular osteotomy and circumferential decompression. Firstly, laminectomy was used to complete the dorsal decompression. Subsequently, the bilateral articular process was removed, and oblique forward inward resection of OPLL anterior intervertebral disk was performed. According to the scope of the upper and lower OPLL, part of the posterior vertebrae bone or even part of 
the pedicle will be removed when it is necessary to "penetrate" on both sides. Finally, the OPLL was pushed forward to the intervertebral space, and OPLL block was removed from the back side to complete the decompression of ventral spinal cord.

Dural defect often occurs in the two steps of the operation: when the laminectomy is performed and when the OPLL block is pushed into the intervertebral space so that it can be separated from dural mater. If there was serious adhesion between ossification block and dural mater, it may also lead to defects of dural. Ventral dural injury or defects was generally not repaired due to surgical field limitations. Dorsal dural injury is first treated with a direct suture with a $5-0$ vessel suture. If sutures are difficult or large dural defects occur, brain cotton would be temporarily used to cover to ensure clear field. After spinal cord decompression, a whole piece of gelatin sponge can be used to cover the dural injury part, without repair of large dural defects. Finally, the surgical incision is closed layer by layer by strict continuous suture.

\section{Diagnostic criteria for CSFL}

The patient is diagnosed as CSFL if their symptoms meet any of the following diagnostic criteria (1) Dural rupture or defect is found during operation, or cerebrospinal fluid outflow is seen; (2) Large amounts (>300 ml/days) of clear or light blood drainage fluid outflow at wound drainage tube remain visible after 48 hours after surgery, and the patients develop severe headaches, nausea, vomiting and other low cranial pressure symptoms.

Postoperative management of CSFL

Once a patient was found to have CSFL, a unified treatment process would be adopted: (1) Continuous lateral position without pillow. The wound negative pressure drainage ball was replaced by atmospheric drainage bag during 12-24 $\mathrm{h}$ after surgery, and the atmospheric drainage bag was placed at the same horizontal position of the surgical incision. (2) Once the patient has symptoms of low intracranial pressure such as headache, the bed tail would be raised for $10 \mathrm{~cm}$, or the trendelenburg position would be adopted to alleviate the symptoms. (3) Water and electrolyte should be taken on a regular basis to strengthen nutritional support. For patients with low intracranial pressure symptoms, intravenous infusion of concentrated sodium chloride was conducted to alleviate the symptoms. (4) The patient should be aware of infection and extend the time of antibiotic use. Antibiotics can be stopped after the drainage tube is removed and the body temperature is normal for 3 consecutive days. (5) The wound drainage tube shall be kept for at least 5 days, but generally not more than 1 week. After the color of the drainage fluid was basically clear, the drainage tube can be removed. One to two stitches were sutured in the whole layer of the drainage tube to avoid the outflow of cerebrospinal fluid from the drainage port.

\section{Predictor of CSFL}

The cases were divided into CSFL group and non-CSFL group based on the CSFL diagnostic criteria. The predictors considered included: (1) Demographic characteristics: age, gender, body mass index; (2) Clinical data: preoperative JOA score, course of disease, complications (hypertension, diabetes), smoking 
history, segment of circumferential decompression (upper, middle, lower thoracic segments), and the number of laminectomy segments. (3) Radiological data: preoperative thoracic CT and MRI were performed in all enrolled cases before surgery. According to the thoracic CT sagittal image, the posterior longitudinal ligament after ossification was divided into three types: isolated, continuous (flat or wavy) and hybrid. The medical personnels would determine whether the segment of circumferential decompression was combined with OLF. The occupying ratio was defined as the OPLL thickness divided by the front and rear diameter of the spinal canal, which can be measured on the sagittal CT scan. The OPLL base ratio was defined as the OPLL width divided by the transverse diameter of the spinal canal, which can be measured on the axial CT scan (Fig. 1). The Intramedullary high signal can be defined in the thoracic MRI T2 weighted image.

\section{Statistical analysis}

This was a retrospective cohort study, SPSS 19.0 (SPSS, USA) statistical software was adopted in this study. The measurement data were expressed with the mean \pm standard deviation. Single-factor regression analysis was used to analyze whether possible predictors had statistical significance. Predictors that are of significance to statistics were included into the multivariate regression analysis. A predictive model was constructed, and a receiver operating characteristic (ROC) curve was drawn.

\section{Results}

\section{Incidence of CSFL}

According to CSFL diagnostic criteria, 19 patients were in the CSFL group, and 42 patients were in the non-CSFL group. The incidence of CSFL of 31.1\%. Among the enrolled cases, 7 are males and 12 are females, with an average age of $49.8 \pm 11.4$ years. Dural injury was found in 14 cases, including 10 ventral dural injury, and 4 dorsal dural injury. Although no dural injury was found in the other 5 cases, they met the diagnostic criteria of CSFL (2) (Table 1). 
Table 1

The comparison of demographic characteristics, radiological and surgical data between CSFL group and non-CSFL group

\begin{tabular}{|c|c|c|c|}
\hline & CSFL group & non- CSFL group & $P$ value \\
\hline Gender(male/female) & $7 / 12$ & $22 / 20$ & 0.26 \\
\hline Age & $49.8 \pm 11.4$ & $55.0 \pm 13.5$ & 0.15 \\
\hline Body mass index $(\mathrm{kg} / \mathrm{m} 2), \mathrm{BMI}$ & $27.6 \pm 4.5$ & $28.0 \pm 5.4$ & 0.81 \\
\hline Duration of disease(month) & $11.2 \pm 10.2$ & $8.3 \pm 7.7$ & 0.22 \\
\hline Preoperative JOA score & $4.8 \pm 1.9$ & $5.3 \pm 1.8$ & 0.35 \\
\hline Diabetes mellitus(Yes/No) & $1 / 18$ & $3 / 39$ & 1.00 \\
\hline Hypertension(Yes/No) & $5 / 14$ & $7 / 35$ & 0.49 \\
\hline Smoking(Yes/No) & $16 / 3$ & $13 / 29$ & $<0.001$ \\
\hline Segment of circumferential decompression & & & 0.001 \\
\hline Upper & 8 & 7 & \\
\hline Middle & 9 & 11 & \\
\hline Lower & 2 & 24 & \\
\hline Type of OPLL & & & 0.21 \\
\hline Beaked & 8 & 25 & \\
\hline Non-Beaked & 11 & 17 & \\
\hline Combined with OLF(Yes/No) & $13 / 6$ & $13 / 29$ & 0.006 \\
\hline Occupying ratio(\%) & $43.4 \pm 7.6$ & $36.1 \pm 6.8$ & $<0.001$ \\
\hline OPLL base ratio(\%) & $65.2 \pm 6.2$ & $56.9 \pm 7.9$ & $<0.001$ \\
\hline Intramedullary high signal(Yes/No) & $3 / 16$ & $6 / 36$ & \\
\hline Operative time $(\mathrm{min})$ & $186.6 \pm 71.0$ & $163.4 \pm 60.8$ & 0.2 \\
\hline Bleeding $(\mathrm{ml})$ & $981.6 \pm 863.2$ & $949.8 \pm 650.0$ & 0.87 \\
\hline Number of laminectomy & $5.9 \pm 3.1$ & $3.7 \pm 2.2$ & 0.01 \\
\hline Time of drainage(day) & $5.6 \pm 1.0$ & $4.2 \pm 1.3$ & $<0.001$ \\
\hline length of stay(day) & $16.3 \pm 6.3$ & $15.8 \pm 6.7$ & 0.77 \\
\hline
\end{tabular}

Complications related to CSFL 
12 cases (63.2\%) had symptoms of low intracranial pressure like headaches, and the symptoms were relieved after drainage tube removal; 1 patient (5.3\%) had cerebrospinal fluid outflow from the incision, and the wound healed successfully after debridement; 1 case $(5.3 \%)$ was re-admitted to the hospital for fever 3 weeks after the operation, and the wound effusion was found to be cured after 2 weeks of antiinfective treatment; No patient had complications such as falling pneumonia, urinary tract infection, central nervous system infection or venous thrombosis of lower extremity. The average retention time of drainage tube was $5.6 \pm 1.0$ days, which was significantly higher than that of non-CSFL group (4.2 \pm 1.3 days, $\mathrm{P}<0.001)$; the average hospitalization days were $16.3 \pm 6.3$ days, which was slightly higher than those in the non-CSFL group $(15.8 \pm 6.7$ days), but there was no statistical difference $(P=0.77)$.

\section{Predictor of CSFL}

Univariate regression analysis showed statistical differences $(P<0.05)$ in smoking history, segment of circumferential decompression, combined with OLF, number of laminectomy, occupying ratio and OPLL base ratio (Table 2). The above variables were included in the Multivariate regression model, and the variable entered the model in the way of "step forward (condition)" when running the statistical software. The resulting Logistic model was statistically significant $\left(\chi^{2}=44.78, P<0.001\right)$. The model can correctly classify $86.9 \%$ of the research object, with a sensitivity of $73.7 \%$, a specificity of $90.5 \%$. Among the six variables, smoking history, segment of circumferential decompression and OPLL base ratio were statistically significant (Table 3). The regression equation was:

$\operatorname{Ln}($ odds $)=-20.09+3.41 \times S+5.24 \times U+4.05 \times M+0.22 \times B$

Where $S$ represents Smoking $(0=$ No, $1=$ Yes $), U$ represents Upper thoracic $(0=$ No, $1=$ Yes $), M$ represents Middle thoracic $(0=$ No, $1=Y e s)$, and $B$ represents Base ratio.

A Hosmer-Lemeshow test method was used to analyze the fitting degree of the model. The results showed that the difference was not statistically significant $(P=0.62)$, indicating that the model fitted well and could be used for risk prediction. The PRE was used as the target variable, the CSFL as the state variable, then a ROC curve was drawn. It was shown that the ROC curve was in the upper left corner (area under the curve $=0.955,95 \% \mathrm{Cl} 0.91-1.00, \mathrm{P}<0.001$ ), indicating good predictability of the model (Fig. 2). youden index $=$ sensitivity + specificity -1 . The maximum Yorden index of this model was 0.81 . This model had a sensitivity of $94.7 \%$ and a specificity of $85.7 \%$. 
Table 2

Univariate Analysis for Predictors of postoperative CSFL

\begin{tabular}{|c|c|c|c|c|c|c|c|}
\hline Predictors & OR & $95 \% \mathrm{Cl}$ & $P$ & Predictors & OR & $95 \% \mathrm{Cl}$ & $P$ \\
\hline age & 0.97 & $\begin{array}{l}0.93- \\
1.01\end{array}$ & 0.16 & duration of disease & 1.04 & $\begin{array}{l}0.98- \\
1.10\end{array}$ & 0.23 \\
\hline sex & 0.53 & $\begin{array}{l}0.17- \\
1.61\end{array}$ & 0.26 & cigarette & 11.90 & $\begin{array}{l}2.95- \\
48.05\end{array}$ & 0.001 \\
\hline BMI & 0.99 & $\begin{array}{l}0.88- \\
1.10\end{array}$ & 0.81 & type of OPLL & 0.50 & $\begin{array}{l}0.17- \\
1.49\end{array}$ & 0.21 \\
\hline JOA score & 0.86 & $\begin{array}{l}0.63- \\
1.18\end{array}$ & 0.34 & combined with OLF & 0.21 & $\begin{array}{l}0.06- \\
0.67\end{array}$ & 0.008 \\
\hline DM & 0.72 & $\begin{array}{l}0.07- \\
7.43\end{array}$ & 0.78 & $\begin{array}{l}\text { number of } \\
\text { laminectomy }\end{array}$ & 1.35 & $\begin{array}{l}1.09- \\
1.67\end{array}$ & 0.007 \\
\hline hypertension & 1.79 & $\begin{array}{l}0.49- \\
8.59\end{array}$ & 0.38 & occupying ratio & 1.15 & $\begin{array}{l}1.05- \\
1.25\end{array}$ & 0.002 \\
\hline CD level & & & & base ratio & 1.15 & $\begin{array}{l}1.06- \\
1.26\end{array}$ & 0.001 \\
\hline upper & 13.71 & $\begin{array}{l}2.35- \\
79.99\end{array}$ & 0.004 & $\begin{array}{l}\text { Intramedullary high } \\
\text { signal }\end{array}$ & 0.89 & $0.20-4.00$ & 0.88 \\
\hline middle & 9.82 & $\begin{array}{l}1.81- \\
53.22\end{array}$ & 0.008 & & & & \\
\hline
\end{tabular}

Table 3

Multivariate Analysis for Predictors of postoperative CSFL

\begin{tabular}{|lccccc|}
\hline \multicolumn{7}{|c}{ B } & Walds & Odds ratio & 95\%Cl & Pvalue \\
\hline Smoking & 3.41 & 8.66 & 30.1 & $3.1-291.2$ & 0.003 \\
\hline Segment of circumferential decompression & & \\
\hline Upper thoracic & 5.24 & 9.25 & 188.0 & $6.4-5494.9$ & 0.002 \\
\hline Middle thoracic & 4.05 & 7.76 & 57.4 & $3.3-993.4$ & 0.005 \\
\hline OPLL base ratio & 0.22 & 7.17 & 1.3 & $1.1-1.5$ & 0.007 \\
\hline
\end{tabular}

\section{Discussion}

CSFL is a common complication of spinal surgery, and the incidence of different sites varies greatly. Hannallah [ reported that the incidence of CSFL after cervical surgery was only $1 \%$. Marcelo [ reported that the incidence of CSFL after lumbar vertebrae surgery was 3.2\%. The incidence of CSFL after thoracic surgery was much higher than those of other parts of the spine, which was reported to be about $20 \% \otimes 40 \%$ [,]. Moreover, the operative approach differed significantly. Hu [ counted 362 cases of thoracic surgery, 
and the overall incidence of CSFL rate was $32.3 \%$. The incidence of anterior decompression (AD), posterior decompression (PD) and circumferential decompression (CD) were $20.5 \%, 31.1 \%$ and $41.8 \%$, respectively. The incidence of CSFL of CD was much higher than other surgical approaches. Takahara [11] reported that the incidence of CSFL after CD was as high as $40 \%$. The posterior approach of CD was a direct decompression, which required the removal of ventral compression of the spinal cord from the rear. As a result, spinal cord occlusion led to narrow space and limited visual field, which was the reason why the incidence of CSFL of circular decompression surgery was higher than that of other approaches. The surgical method in this study is CD, and the incidence of CSFL was $28.0 \%$, which was slightly lower than the previous result. The improvement of surgical instruments is an important factor. During the operation, we used the piezosurgery to assist in spinal cord decompression. The safety and efficiency of piezosurgery in the removal of bone structure have been recognized by academic circles. Compared with the traditional bone knife or high-speed drill, high-energy, high-frequency but low-amplitude characteristics can achieve basically no vibration when removing ossified blocks, and the characteristics of "cutting soft and not cutting hard" can help to avoid dural injury. As a result, the rational use of piezosurgery can effectively reduce the incidence of CSFL. But it must be admitted that even if the incidence of CSFL could be reduced through operator's surgical techniques and improved surgical instruments, nearly a third of cases were diagnosed with CSFL. Therefore, predicting the related factors that can easily lead to CSFL before surgery and screening out the high risk population in thoracic OPLL circumferential decompression was helpful to warn the operator and inform patients of the risk in advance.

At present, there are few reports of exploring predictors of CSFL after thoracic OPLL circumferential decompression, and there are no universally-acknowledged conclusions. Sun $[$ found that patients with more than 3-segment laminectomy (odds ratio $=2.4$, $\mathrm{P}$ odds ratio $=0.01$ ) have higher incidence of $\mathrm{CSFL}$ (odds ratio $=2.4, P<0.01$ ). In this study, although the number of removed vertebral plates in the CSFL group was significantly higher than in the non-CSFL group (5.9 VS 3.7, P=0.01), there was no statistical difference in logistic regression analysis. This was related to differences in the etiology and surgical methods of the two studies: Sun studied the cases with OLF, the surgical method was posterior approach laminectomy and decompression, and the dural injury occurred only in the dorsal spinal cord. By contrast, in this study, the included case was OPLL, the surgical method was circumferential decompression. Dural injury occurred more often in the ventral spinal cord. As a result, the factors leading to CSFL were more complex. Nicholas \ found that patients with diabetes $(O R=2.3, P=0.04)$ or smoking history $(O R=3.4, P$ $=0.02$ ) were more likely to develop CSFL in the study of dural injury during lumbar surgery. The smoking history in this study is also a risk factor for the CSFL after thoracic OPLL circumferential decompression $(\mathrm{OR}=30.1, \mathrm{P}=0.003)$, which was consistent with the study by Nicholas. It is widely believed that smoking can lead to thinning of the fascia and decreased toughness [. The dura mater, though different from the abdominal pelvic fascia, has a similar composition and structure. It can therefore partly explain why smoking increases the risk of CSFL. Hu [12] found that CSFL is more likely to occur in T5-7 circular decompression surgery than other segments. In our study, the probability of CSFL occurring in the upper thoracic segment $(O R=188.0, P=0.002)$ and the middle thoracic segment $(O R=57.4, P=0.005)$ was also 
significantly higher than that of the lower thoracic segment. This is mainly because the middle and upper thoracic segments were narrower and had limited surgery space, so they were likely to cause dural injury.

The shape of OPLL itself may also be the cause of postoperative CSLF. The sagittal and transverse diameters of OPLL were used to evaluate the occupying ratio and OPLL base ratio. It was found that the vertebral occupying ratio and OPLL base ratio in the CSFL group were higher than those in the non-CSFL group. The multi-factor regression analysis showed that the OPLL base ratio was a predictor of postoperative CSFL. The wider the OPLL base, the more likely postoperative CSFL (or $=1.3, P=0.007$ ) will happen. Du [ came to a similar conclusion in studying the risk factors of CSFL after anterior approach cervical OPLL decompression. Du collected the data of 90 patients who used anterior approach cervical decompression to treat cervical OPLL, and found that CSLF is more likely to happen during resection of wide-base OPLL $(O R=1.09, P=0.012)$ than that of narrow-base OPLL. The reason was that the base at both head and tail of OPLL was cut off in advance in order to take out the OPLL ossific block. As a result, the wide base of OPLL will increase the difficulty of resection, cause more dural invasion, and develop CSLF. After cutting the base at both head and tail of OPLL, we used the "collapse method" to push the free OPLL ossific block directly to the anterior vertebral gap without directly contacting the final part of the OPLL ossific block near the midline. Therefore, the OPLL sagittal diameter had little effect on the formation of CSFL, which was consistent with the results of regression analysis in this paper.

As far as I know, there is no literature on the predictive model of CSFL after thoracic circumferential decompression. This study attempted to establish a predictive model of CSFL probability after thoracic OPLL circumferential decompression. The Logistic regression analysis found that smoking history, segment of circumferential decompression and OPLL base were statistically significant, which can be used to predict the occurrence probability of postoperative CSFL. In general, the area under the ROC curve $>0.6$ indicates that the model has good predictability. The ROC of the model was 0.955 , indicating that the model can well predict the risk of CSFL after thoracic OPLL circumferential decompression. There are still some shortcomings in this study: (1) Because the focus of this study is to explore the predictive model of CSFL, patients were not followed up for long periods. So the long-term effects of CSFL on patients, such as pseudocyst formation, nerve function recovery and so on, require further follow-up studies; (2) The number of cases was limited, which was limited by the lower incidence of thoracic OPLL, and not all cases require circumferential decompression, so long-term accumulation of cases is required.

\section{Conclusions}

To sum up, the predictive model established in this study has a high predictive effect. When the patients with thoracic OPLL have smoking history or the segment of circumferential decompression is located in the upper or middle thoracic spine or the OPLL has a wide base, the surgeon should be highly alert to the possibility of postoperative CSFL and warn the patient before surgery.

\section{Abbreviations}


CSFL

cerebrospinal fluid leakage

OPLL

ossification of posterior longitudinal ligament

OLF

ossification of the ligamentum flavum

ROC

receiver operating characteristic

$A D$

anterior decompression

PD

posterior decompression

\section{Declarations}

Acknowledgements

CD: circumferential decompression

There was no conflict of interest by any of the authors including financial and personal relationships with other people or organizations that inappropriately influenced this study.

Authors' contributions

ZJ collected, analyzed, and interpreted the data and wrote the manuscript. WBT, CZQ performed the surgery, designed the protocol, revised the manuscript. All the authors have read and approved the final manuscript.

Funding

No funding

Availability of data and materials

The datasets used and/or analysed during the current study are available from the corresponding author on reasonable request.

Ethics approval and consent to participate

The experimental protocol was established, according to the ethical guidelines of the Helsinki Declaration and was approved by the Human Ethics Committee of Peking University International Hospital. Written informed consent was obtained from each participant.

Consent for publication 
Written informed consent for publication was obtained from each participant.

Competing interests

All authors declare that they have no conflict of interest.

\section{References}

1. Matsumoto M, Chiba K, Toyama Y, et al. Surgical results and related factors for ossification of posterior longitudinal ligament of the thoracic spine: a multi-institutional retrospective study [J]. Spine (Phila Pa 1976), 2008, 33(9):1034-1041.

2. Ma X, Howard S. An, Zhang $Y$, et al. A radical procedure of circumferential spinal cord decompression through a modified posterior approach for thoracic myelopathy caused by severely impinging anterior ossification [J]. The Spine Journal, 2014, 14(4):651-658.

3. Xu Z, Hu Y, Sun C, et al. Treatment for Thoracic Ossification of Posterior Longitudinal Ligament with Posterior Circumferential Decompression[J]. Orthopaedic Surgery. 2017, 9(2): 206-214.

4. Yamazaki M, Mochizuki M, Ikeda Y, et al. Clinical results of surgery for thoracic myelopathy caused by ossification of the posterior longitudinal ligament: operative indication of posterior decompression with instrumented fusion [J]. Spine (Phila Pa 1976), 2006, 31(13): 1452-1460.

5. Li M, Meng H, Du J, et al. Management of thoracic myelopathy caused by ossification of the posterior longitudinal ligament combined with ossification of the ligamentum flavum - A retrospective study[J]. Spine J 2012, 12(12): 1093-1102.

6. Khan MH, Rihn J, Steele G, et al. Postoperative management protocol for incidental dural tears during degenerative lumbar spine surgery: A review of 3,183 consecutive degenerative lumbar cases[J]. Spine (Phila Pa 1976) 2006, 31(22): 2609-13.

7. Hannallah $D$, Lee J, Khan $M$, et al. Cerebrospinal fluid leaks following cervical spine surgery [J]. J Bone Joint Surg Am, 2008, 90(5): 1101-1105.

8. Galarza M, Gazzeri R, Alfaro R, et al. Evaluation and management of small dural tears in primary lumbar spinal decompression and discectomy surgery [J]. J Clin Neurosci, 2018, 50(3):177-182.

9. Kawahara N, Tomita K, Murakami H, Hato T, Demura S, Sekino Y, et al. Circumspinal decompression with dekyphosis stabilization for thoracic myelopathy due to ossification of the posterior longitudinal ligament [J]. Spine (Phila Pa 1976) 2008, 33(1): 39-46.

10. Hu P, Yu M, Liu X, Liu Z, Jiang L. A circumferential decompression-based surgical strategy for multilevel ossification of thoracic posterior longitudinal ligament [J]. Spine J 2015, 15(12): 24842492.

11. Takahata $\mathrm{M}$, Ito $\mathrm{M}, \mathrm{Abumi} \mathrm{K}$ et al. Clinical results and complications of circumferential spinal cord decompression through a single posterior approach for thoracic myelopathy caused by ossification of posterior longitudinal ligament [J]. Spine (Phila Pa 1976), 2008, 33(11): 1199-1208. 
12. Hu P, Yu M, Liu X, et al. Cerebrospinal Fluid Leakage after Surgeries on the Thoracic Spine: A Review of 362 Cases [J]. Asian Spine Journal, 2016, 10(3): 472-479.

Sun X, Sun C, Liu X, et al. The frequency and treatment of dural tears and cerebrospinal fluid leakage in 266 patients with thoracic myelopathy caused by ossification of the ligamentum flavum [J]. Spine (Phila Pa1976), 2012, 37(12): E702-707.

13. Nicholas Ahn, Uri Ahn, Zachary Post, et al. Smoking, diabetes and excessive preoperative epidural steroid administration are risk factors for intraoperative dural tears. Proceedings of the NASS 19th Annual Meeting / The Spine Journal, 2004, 4: 3S-119S.

14. Goyal DKC, Divi SN, Bowles DR, et al. How Does Smoking Influence Patient-reported Outcomes in Patients After Lumbar Fusion [J]? Clin Spine Surg, 2021, 34(1): E45-E50.

15. Du YQ, Duan WR, Chen Z, et al. Risk Factors and Management of Dural Defects in Anterior Surgery for Cervical Ossification of the Posterior Longitudinal Ligament. World Neurosurg. 2018, 111(3): e527-e538.

\section{Figures}

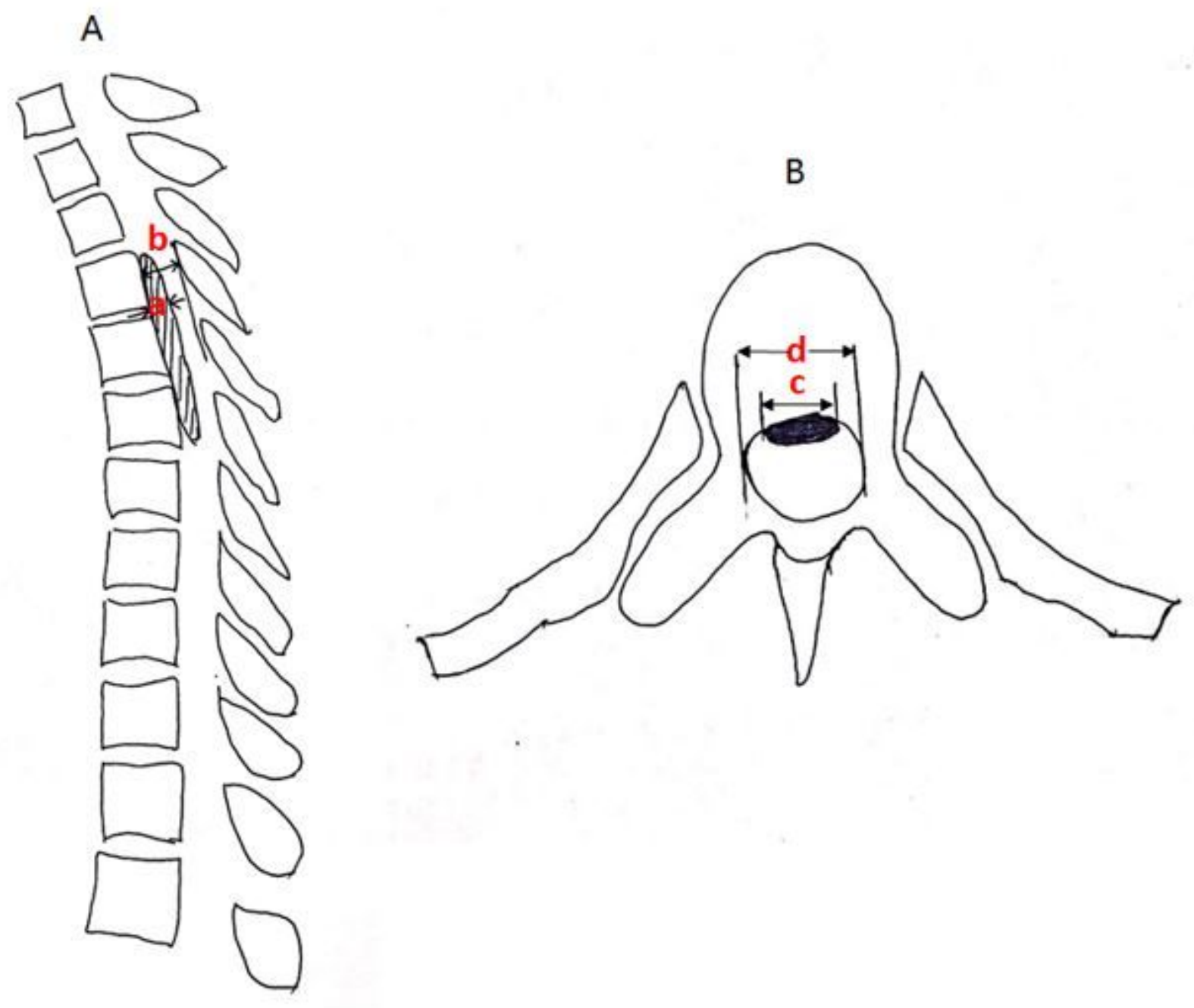


Figure 1

Measurement methods for the occupying ratio and OPLL base ratio. A: The occupying ratio was defined as the OPLL thickness divided by the front and rear diameter of the spinal canal, which can be measured on the CT scan $(a / b * 100 \%)$. B: The OPLL base ratio was defined as the OPLL width divided by the transverse diameter of the spinal canal on the axial CT scan $\left(\mathrm{c} / \mathrm{d}^{*} 100 \%\right)$

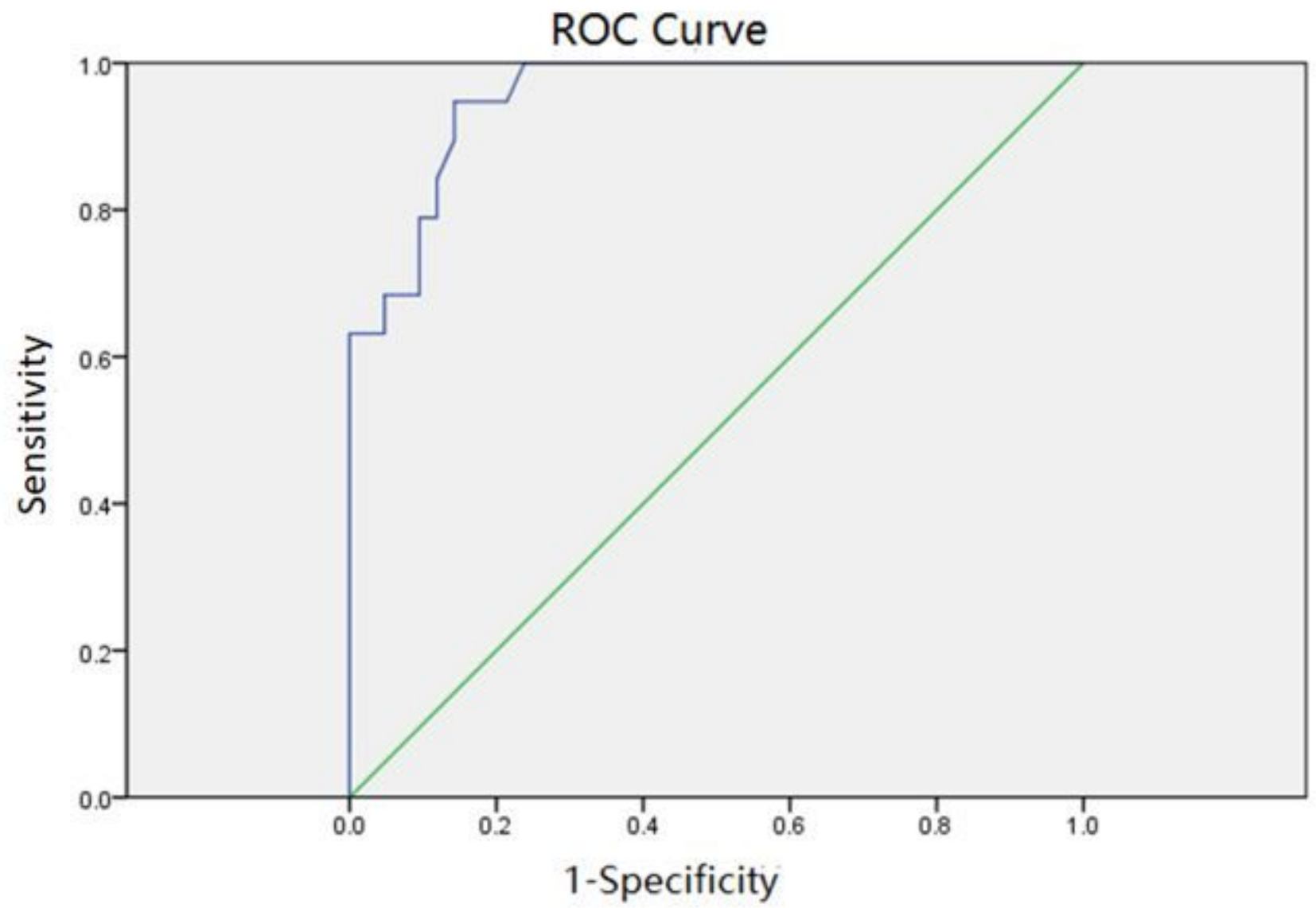

Figure 2

ROC curve showing the overall ability of the model to classify CSFL. The area under the curve $=0.955$. Typical case (Figure 3) 

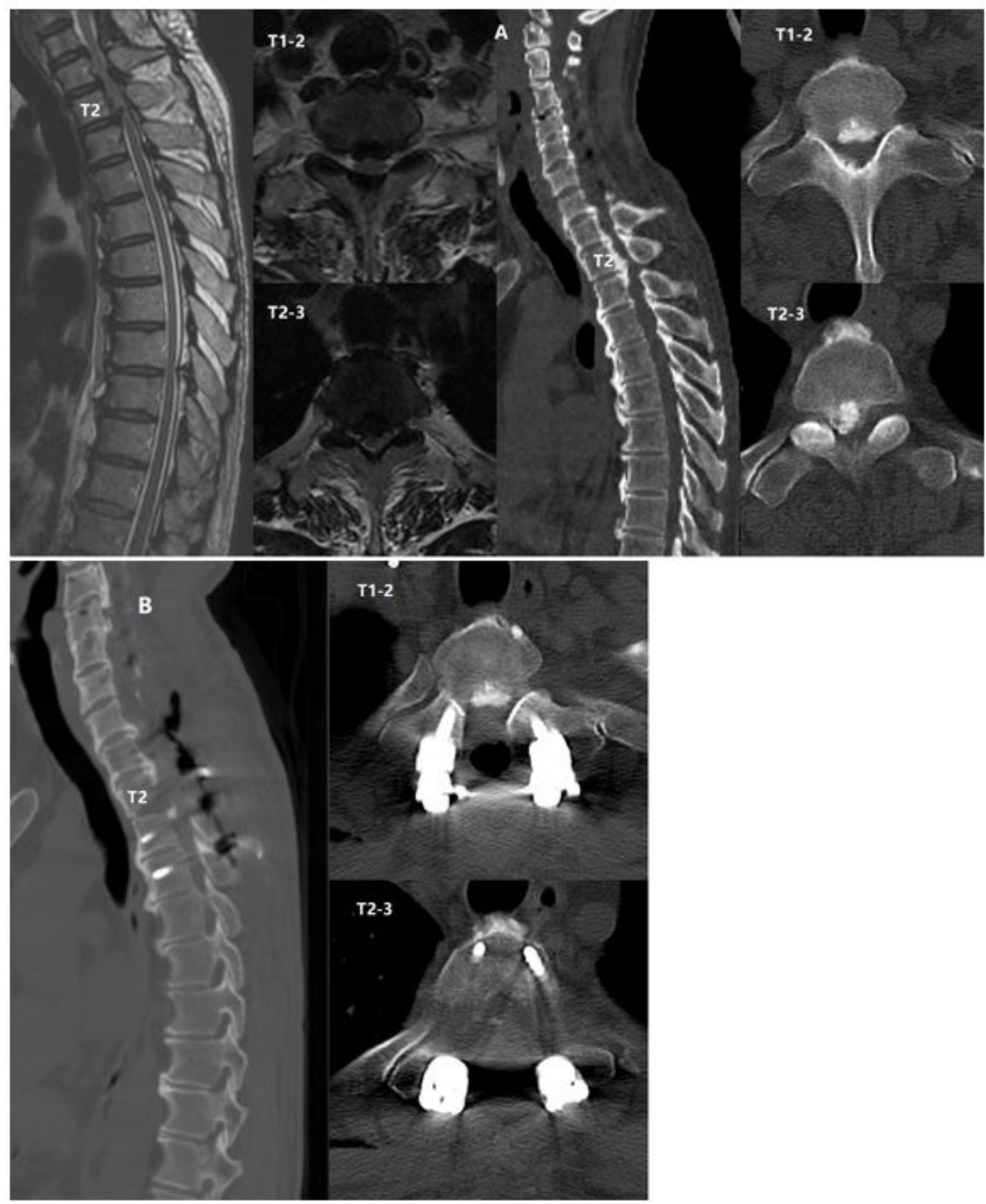

\section{Figure 3}

A 60 years old man presented with progressive exacerbation of lower limb weakness and walking instability for 6 months. Pre-operative JOA score was 3. A: The CT and MRI scans both showed continuous OPLL from T1 to T3 with the worst compression at T2-3. B: The patient underwent T1-3 laminectomy and selectively T2-3 circumferential decompression. Postoperative CT scans showed that 
OPLL was completely removed at T2-4 and retained in the rest regions. The JOA score was 9 in the follow-up of one year and the recovery rate was $75.0 \%$. 